\title{
Trifluoroacetyl as an Orthogonal Protecting Group for Guanidines
}

Sandra Bartoli, Kim B. Jensen and Jeremy D. Kilburn*

Department of Chemistry, University of Southampton, Southampton, SO17 1BJ, U.K.

Table of contents:

1. General methods S2

2. Abbreviations S4

3. Materials S5

4. References S6 
General Methods. Commercially available compounds were used without further purification. When necessary solvents were dried according to literature procedures ${ }^{[1]}$. Peptide and library synthesis on solid phase were performed in glass vessels with sinter frits or polypropylene filtration tubes with polyethylene frits on a Visiprep SPE Vacuum Manifold (Supelco). Reaction vessels were agitated either on a shaker (Stuart Scientific Flash Shaker SF1) or on a blood tube rotator (Stuart Scientific Blood Tube Rotator SB1). Thin layer chromatography (TLC) was performed on aluminium-backed plates silica gel 60 F254. Column chromatography was performed on 40-60 mesh silica. All melting points were determined in open capillary tubes using a Gallenkamp Electrothermal Melting Point Apparatus and are uncorrected. Optical rotations were measured on a PolAAr-2001 Polarimeter. Proton NMR spectra were obtained at $300 \mathrm{MHz}$ on a Bruker AC 300 and at $400 \mathrm{MHz}$ on a Bruker DPX 400. Carbon NMR spectra were recorded at $75 \mathrm{MHz}$ on a Bruker AC 300 and at $100 \mathrm{MHz}$ on a Bruker DPX 400. Chemical shifts are reported in ppm on the $\delta$ scale relatively to the signal of the solvent used. Coupling constants are given in Hz. Mass spectra were obtained on a VG analytical 70-250-SE normal geometry double focussing mass spectrometer. All electrospray (ES) spectra were recorded on a Micromass Platform quadrupole mass analyser with an electrospray ion source using acetonitrile or methanol as solvent. MALDI-TOF spectra were recorded on a Dynamo 
DE-linear-MALDI-TOF mass spectrometer. UV absorbance of ninhydrin and Fmoc assays were measured on a Hewlett-Packard 8452A Diode Array spectrometer using two way quartz cells. Absorbance values were recorded at $570 \mathrm{~nm}$ (ninhydrin) and $302 \mathrm{~nm}$ (Fmoc). 
Abbreviations: Aloc, allyloxycarbonyl; Boc, tert-butyloxycarbonyl; CBS, carboxylate binding site; Cbz, benzyloxycarbonyl; DIC, $N, N^{\prime}-$ diisopropylcarbodiimide; DBU, 1,8 diazabicyclo[ 5.4.0] undec-7-ene; Ddpe, 1-(4,4-dimethyl-2,6-dioxocyclohex-1-ylidene)phenylethyl; DIPEA, disopropylethylamine; DMAP, 4-(dimethylamino)pyridine; DMF, N, N-dimethylformamide; DMS, dimethylsulfide; DMSO, dimethyl sulphoxide; EDC, 1-(3-dimethylaminopropyl)-3-ethylcardodiimide hydrochloride; EDT, ethanedithiol; FC, flash chromatography; Fmoc, 9-fluorenyloxycarbonyl; HOBt, 1-hydroxybenzotriazole; PyBOP, benzotriazole-1-yl-oxy-tris-pyrrolino-phosphonium hexafluorophosphate; TFA, trifluoroacetic acid; TFAC, trifluoroacetyl; TIS, triisopropyl silane. 
Materials. (2-Amino-ethyl)-carbamic acid tert-butyl ester, (3Amino-propyl) carbamic acid tert-butyl ester, (3-Amino-propyl) carbamic acid benzyl ester (1), (2-Isothiocyanatoethyl) carbamic acid allyl ester, (2-Isothiocyanatoethyl) carbamic acid tert-butyl ester, (3-Isothiocyanatopropyl) carbamic acid tert-butyl ester (2) were synthesised according to literature procedures ${ }^{[2,3]}$.

\section{References}

[1] Perrin D. D., Armarego W. L. F., Purification of Laboratory Chemicals, Pergamon press, Oxford, (3rd Ed) 1988.

[ 2] a) Kneeland D. M., Ariga K., Lynch V. M., Huang C.-Y., Anslyn E. V., J.Am. Chem. Soc. 1993, 115, 10042-10055;

[3] Jensen, K. B.; Braxmeier, T. M.; Demarcus, M.; Frey, J. G.; Kilburn, J. D. Chem. Eur. J. 2002, 8, 1300-1309. 